\title{
Water-lubricated bearings of ship propeller shafts - problems, experimental tests and theoretical investigations
}

\author{
Wojciech Litwin, Ph. D. \\ Gdansk University of Technology
}

\begin{abstract}

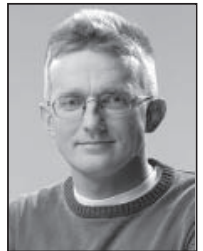

In recent years can be met ships whose propeller shaft polymer bearings are lubricated with water. It results from simplicity and associated low cost of such solution which is also environmentally friendly as no risk of pollution is involved. However the solution is not free of disadvantages. The main problem is intensive wear of bush material, occurring in certain cases. It very often results from errors of improper design and machining and mounting operations. Another problem is a limited value of its hydrodynamic load-carrying capacity, resulting from low viscosity of water used as a lubricating medium. As results from the performed research investigations, bearing of the kind is a highly sensitive unit. Problems of choosing a suitable bearing clearance, designing an optimum bush geometry, selecting a proper bush material are crucial for lifitime of the bearing.
\end{abstract}

Keywords: bearing; water-lubricated bearings; propeller shaft bearing

\section{INTRODUCTION}

Increasing ecological awareness, more and more stringent requirements for environmental protection, fines for emission of noxious substances to sea, and economical calculations have resulted in that shipowners are searching for inexpensive, simple and reliable design solutions for building new ships and modernizing existing ones. These are main reasons for which slide bearings fitted with polymer water-lubricated bush are more and more willingly applied to propeller shaft bearing. Similar reasons are decisive of that water-lubricated bearings are more and more often applied also in water power plants and pumps in which a pumped liquid, usually water, serves as a lubricating medium. They find application also in mining industry, water conditioning stations or land melioration systems.

Worldwide increasing demands concerning durability and reliability make that more and more stringent requirements for bearings are introduced. As results from experience of classification institutions and shipyards, well designed and assembled water-lubricated bearings are able to operate correctly for more than ten years. Unfortunately from the collected data it also results that many failures occur each year on ships, especially in high-loaded propeller shaft bearings. Such failures greatly endanger sea navigation as they may lead to ship disablement. In situation of the kind, when oil serves as a lubricating medium, its leakage results in pollution of sea water. If ship's crew and owner do not immediately decide on docking and repairing the ship its serious failure will be possible to happen (Fig. 1).

Water-lubricated bearing of ship propeller shaft, like most materials and components for building and outfitting the ships, should be approved by a classification institution. Many kinds of polymer materials for bearings, applicable in shipbuilding, are present on the market today. For instance the list issued by Lloyd's Register of Shipping contains over a hundred kinds of such materials produced in many countries worldwide [1].

Producers of bearing materials, in their catalogues and internet pages, very often present a recommended way of selecting a definite material, bearing clearance as well as possible location of lubricating grooves. Therefore the designing of a slide bearing seems very simple. However as results from experience the methods are not always effective. In certain cases an excessive wear of bushes can happen within a short period. Sometimes fast progressing wear results from its operation in mixed friction conditions. It is very hard to obtain fluid friction in a bearing as water film load-carrying capacity is limited due to low viscosity of water. In spite of that water-lubricated bearings are often used in practice. Their lower price as compared with that of oil-lubricated bearings is not without any importance. 

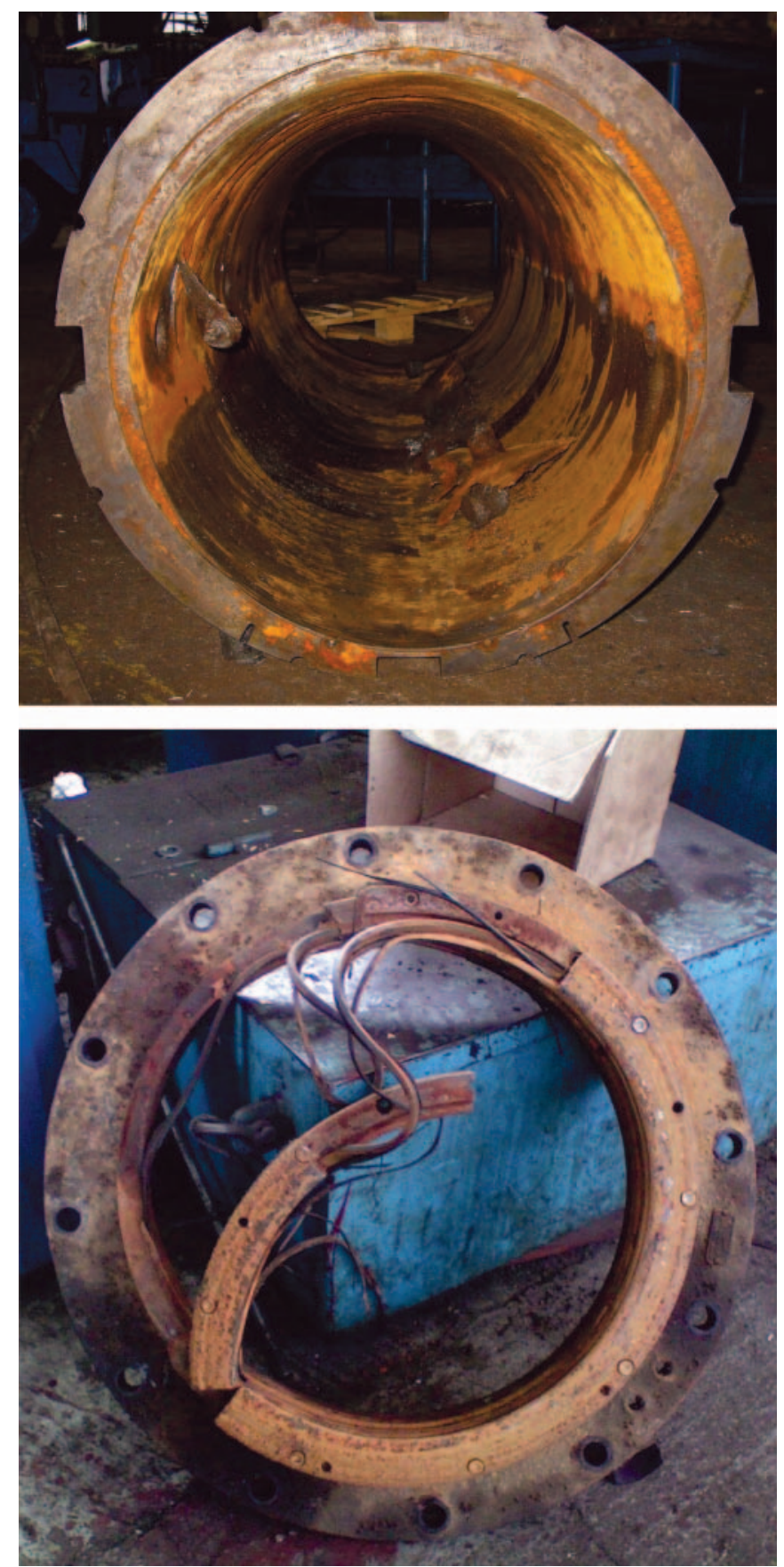

Fig. 1. Completely damaged oil-lubricated slide bearing and sealing of ship propeller shaft

\section{TYPES OF BEARINGS}

Range of the materials available today on the market is very wide. The materials approved for shipbuilding applications can be divided into the three groups: homogenous polymers, composites and rubber which is often substituted by nitrilebutadiene rubber.

Each of them is characteristic of different properties and, resulting from them, advantages and disadvantages. Rubber effectively damps vibrations and is not sensitive to contamination, which is the reason for using it in e.g. cantilever open bearing systems (Fig. 2). It is important that contaminations which fell into rubber bearing accelerate its wear process only moderately. This concerns also hard contaminations, e.g. high-silica sand grains.

Homogenous polymers are reletively elastic. Their modulus of rigidity is usually contained within the range from 800 to

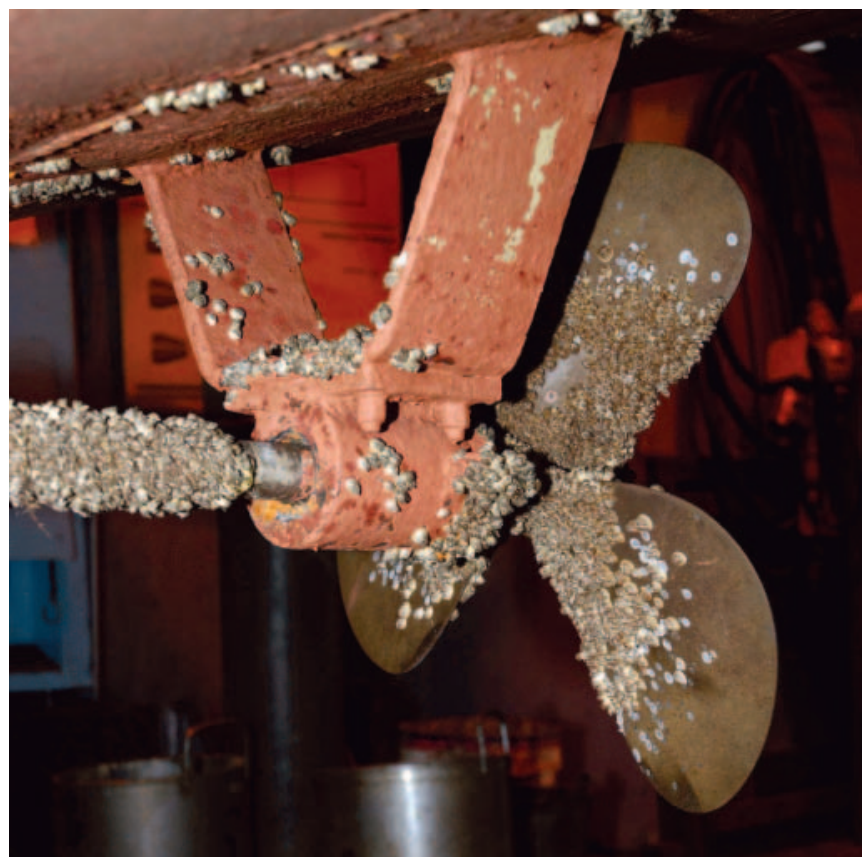

Fig. 2. Cantilever open bearing of yacht's propeller shaft
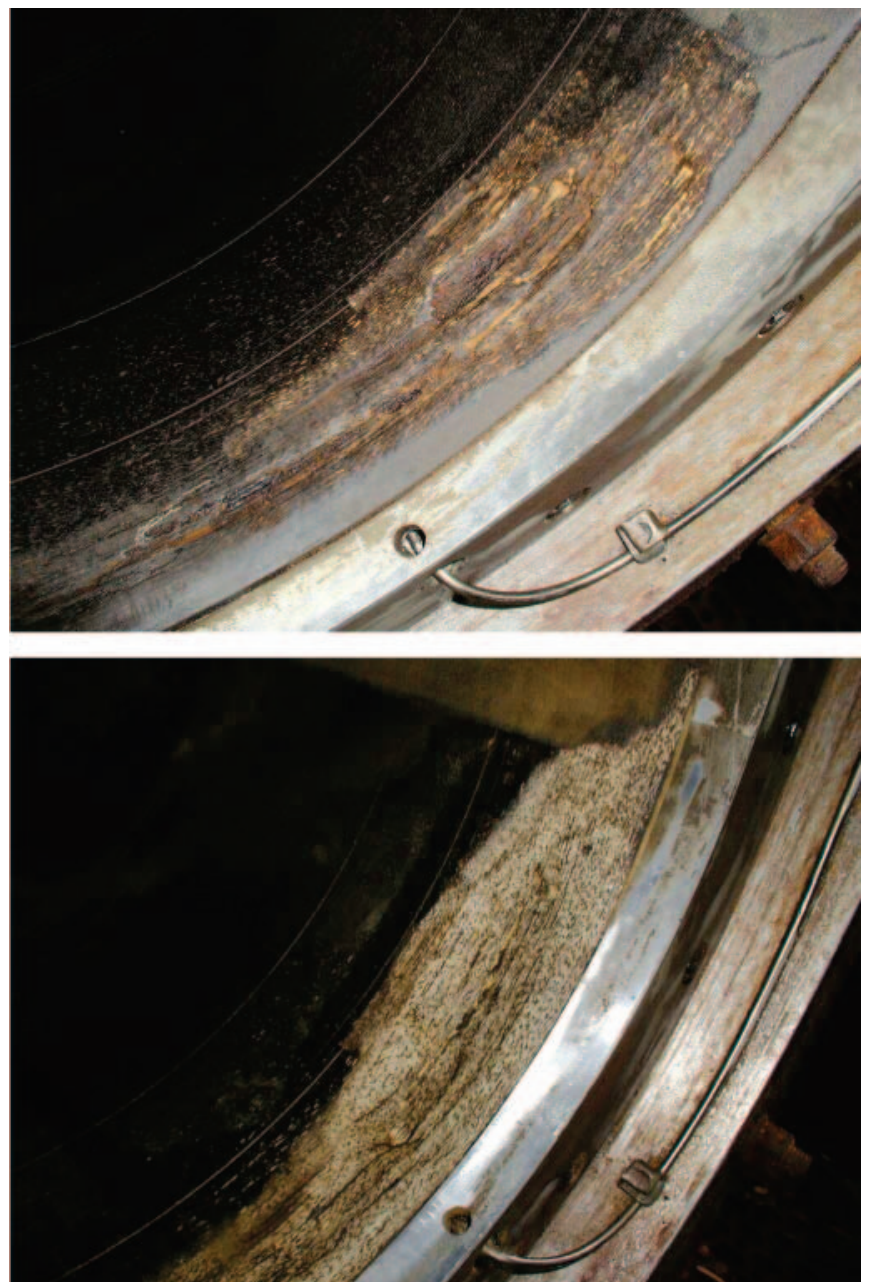

Fig. 3. Damage of a composite bush, resulting from edge thrust resulting from skew position of shaft axis (up); during repair the damaged edge was grinded to avoid cracks and delamination of the composite (down)

$2500 \mathrm{MPa}$. Owing to that such bush is capable of damping shaft vibrations. Polymers, like rubber, relatively well tolerate errors in alignment of shaft axis against bush axis. The thing is in distribution of shaft - to - bush pressure over an extended area as a result of elastic deformation of bush. 
Composites, out of all the above mentioned materials, are characteristic of the largest value of rigidity modulus amounting to about $4500 \mathrm{MPa}$. Therefore they are sensitive to edge thrust in the case of shaft skewing. (Fig. 3), but they also have important merits. They are durable and highly resistant to form distortion which is a disadvantage of some homogenous polymer materials.

The scientific investigations have been so far conducted worldwide justify that ceramics is a good material for bearings operating in water [2]. Slide bearing fitted with ceramic bush can operate in fluid friction conditions at a very small thickness of lubricating film. On the other hand such bearings are especially sensitive to errors in relative position of shaft against bush, particularly to non-parallelism of their axses.

\section{MANUFACTURING AND MOUNTING OF BUSHES}

Bushes are usually manufactured by machine cutting a prefabricate - as a rule - a thin-walled pipe. It is only an outwardly simple operation. In practice it turns out that obtaining a demanded form and accuracy is difficult or completely impossible. Elastic bush material undergoes deformations during machine cutting. In consequence, an inaccuracy of form appears. Therefore it is recommended

\section{$[\mu \mathrm{m}]$}

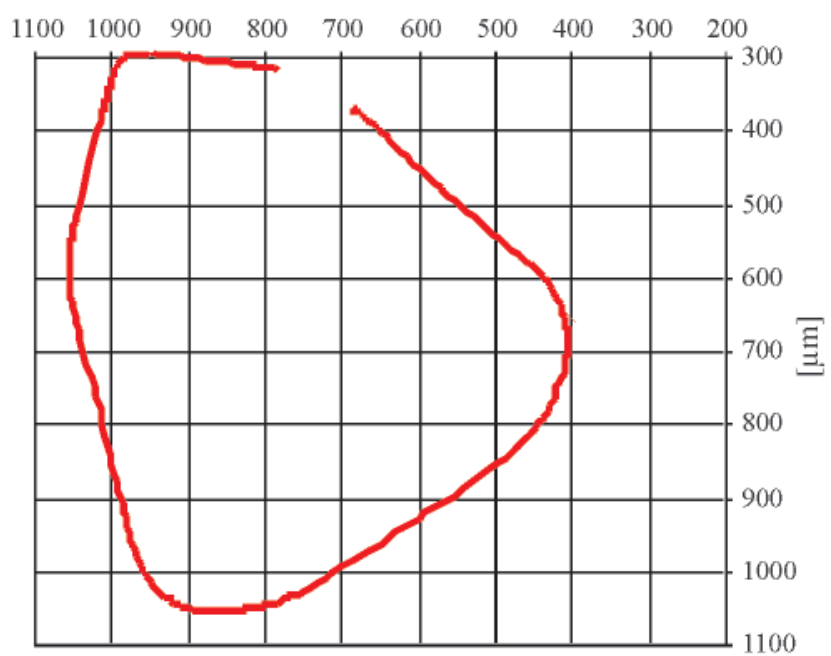

$[\mu \mathrm{m}]$
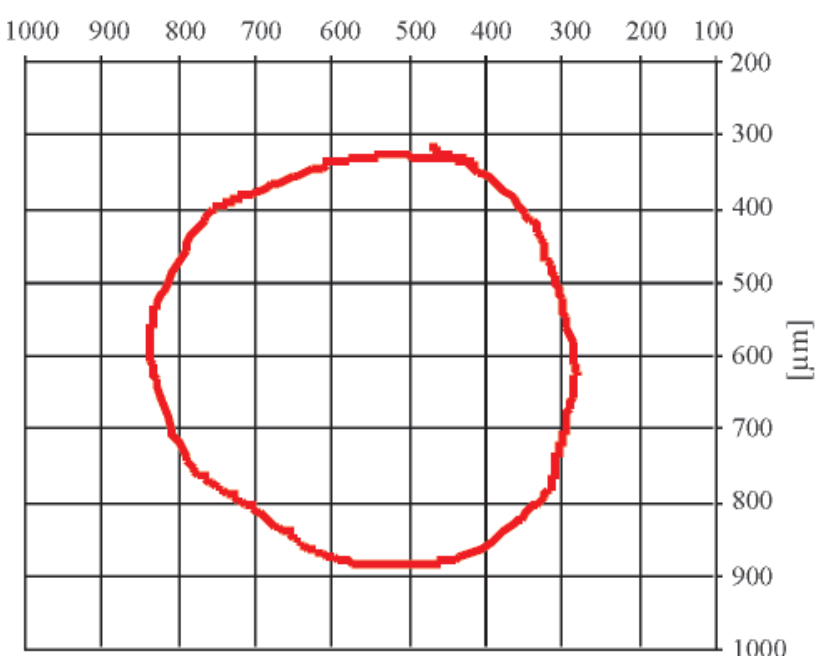

Fig. 4. Circles of gaps measured on both sides of a rubber bearing after its mounting in steel sleeve. Its form errors, especially lack of circularity, can be observed to machine sliding surface of bearing after fixing its bush in a metallic or stiff composite sleeve. Usually a finished bush is ordered from producer who is usually better equipped with technological means to ensure an appropriate machining accuracy. Then the problem of proper mounting is only left for solving. As a rule the forcing method is not recommended as it may lead to bush defromation. Usually the contraction method is applied. The bush is cooled-down to a low temperature by using liquid nitrogen and next mounted into the sleeve. Unfortunately such method does not guarantee the highest accuracy hence bearing clearance may often differ from a design value. Additionally, if longitudinal lubricating grooves are made in the bush a danger of sliding surface deformation arises as the largest deformations appear usually in areas of the smallest thickness of the bush, i.e. in the vicinity of the lubricatineg grooves. Errors in manufacturing and mounting very often cummulate, which results in that bearing geometry differs significantly from the initially designed (Fig. 4). As can be observed from the below given diagrams of measured gap circle, significant deformations have appeared after mounting the rubber bearing of $100 \mathrm{~mm}$ diameter into the steel sleeve. Cylindricity and cirularity errors are found significant. Hence it may be expected that in such bearing an intensive wearingin process would happen and possible hydrodynamic loadcarrying capacity would be very limited.

As results from experience, bearings which practically should be regenerated or replaced are often allowed to be put into operation: for instance a rubber propeller shaft bearing of $385 \mathrm{~mm}$ diameter, in which radial gap was in the range from $4 \mathrm{~mm}$ to almost $10 \mathrm{~mm}$. In the bearing also shaft journal was subjected to wear, that is typical for rubber bearings.
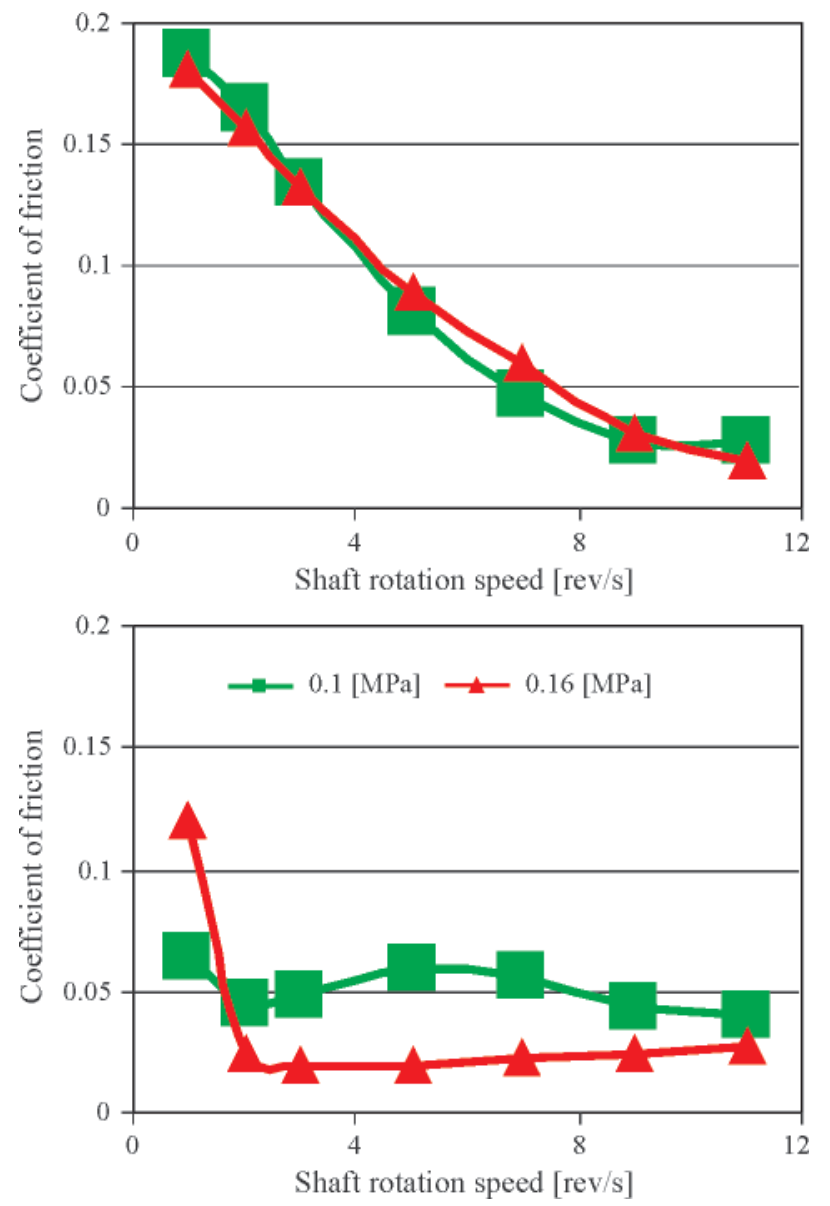

Fig. 5. Diagrams of friction coefficient values in function of shaft rotational speed; up side - the diagram recorded just after mounting the bearing, down side - the diagram obtained after several hours of running-in the bearing 
Another technological problem is the achieving of possible high smoothness of co-operating slide surfaces. It is crucial because minimum thickness of lubricating film and hydrodynamic load-carrying capacity of bearing depends on surface roughness height. In the subject-matter literature publications dealing with influence of height and distribution of roughness on operation of hydrodynamic bearing, can be found. However as results from the author's experimental tests, this is bush roughness height which crucially influences hydrodynamical features of bearing $[3,4]$. The performed tests showed that running-in process of such bearing was very fast and a significant smoothness of slide surfaces was already reached after a few hours of operation. As a result, fluid friction state of operation was achieved faster (Fig. 5).

\section{OPERATIONAL PROBLEMS}

Ship water-lubricated bearings can have different forms recommended by their manufacturers. Such differences concern both values of recommended clearance in bearings and magnitude and number of longitudinal lubricating grooves and their suggested location as well. For fear of clamping the bush material exposed to water some producers recommend to increase clearance values. Most of recognized producers of ship slide bearings recommends to make certain number of lubricating grooves led along the bush. They play a significant role. Due to them, cooling water flow rate increasing is possible. It has a great importance as polymer bush practically makes heat transfer from friction zone to housing impossible. Additionally, the grooves, especially in the systems of open cantilever sea-water-cooled bearings, make it possible to more effecitvely remove wear products and contaminations from the bearings (Fig. 6).

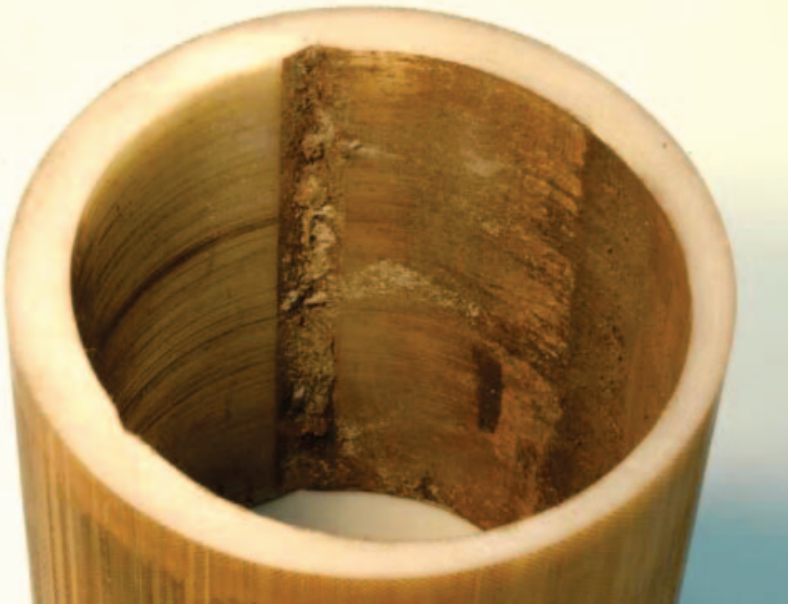

Fig. 6. An unsealed sea-water-lubricated bearing which was exposed to pollution and fouling

Lifetime of ship main shaft bearing depends on many factors. Tribological features of bush material are of a great importance. However to extend lifetime of a bearing significantly it should be so designed as to make it operating in fluid friction regime. As results from the performed tests it is possible to achieve despite water has much lower viscosity than that of lubricating oils [5]. Unfortunately, in consequence, lubricating film thickness is often close to its minimum value. Bearings usually have minimum margin of hydrodynamic load - carrying capacity, and in situations of increased load due to e.g. ship motion in waves, they may operate in mixed friction regime.

Experimental tests showed that even if a high load is imposed on a bearing its operation in fluid friction regime is possible. It results generally from fast process of bush material smoothing and its operation in fluid friction regime at minimum thickness value of lubricating film. Wrong geometry of slide bearing has definitely detrimental influence on its hydrodynamic features. To excessively increase bearing clearance is not recommended. To locate lubricating grooves in lower part of bush is also not recommended, as they make hydrodynamic pressure developing impossible, and, in consequence, they limit load-carrying capacity of the bearing.

As results from a damage analysis of various bearings of the kind, they sufferred failures despite they were correctly designed and manufactured with the use of high-quality materials coming from recognized producers. Reasons of that have been found different. Fast excessive wear resulted sometimes from lack of water flow through bearing due to clogging-up lubricating grooves or faults of crew members who did not put lubricating system circulation pump into operation. In most cases it was changeable heavy operation conditions of ship shaftline, which led to fast progressing wear or even failure of the bearings.

Changeable operational conditions are caused by a few factors. To the most important belong ship's hull elastic structural deformations resulting from ship's motion in waves. During ship motion in heavy waves mass forces applied to shaftline transverse bearings can be risen by dynamic components resulting from ship roll and heave motions. Sometimes loads exerted to bush may be enlarged by dynamic loads due to unbalanced motion of propeller or vibrations generated by its blades passsing through non-homogenous pressure field.

Appropriate alignment of shaftline and bushes is the next important factor which directly influences lifetime of bearings; therefore mounting accuracy is of a special importance. In the case when mounting errors happen the skewing of shaft axis versus bush axis may occur. As a result load-carrying capacity of the bearing will be limited. In the extreme case the so-called edging phenomenon and associated fast progressing wear can appear because values of allowable contact pressure are locally exceeded. Bearings fitted with rigid ceramic, metallic or composite bushes are especially sensitive to the phenomenon (Fig.7).

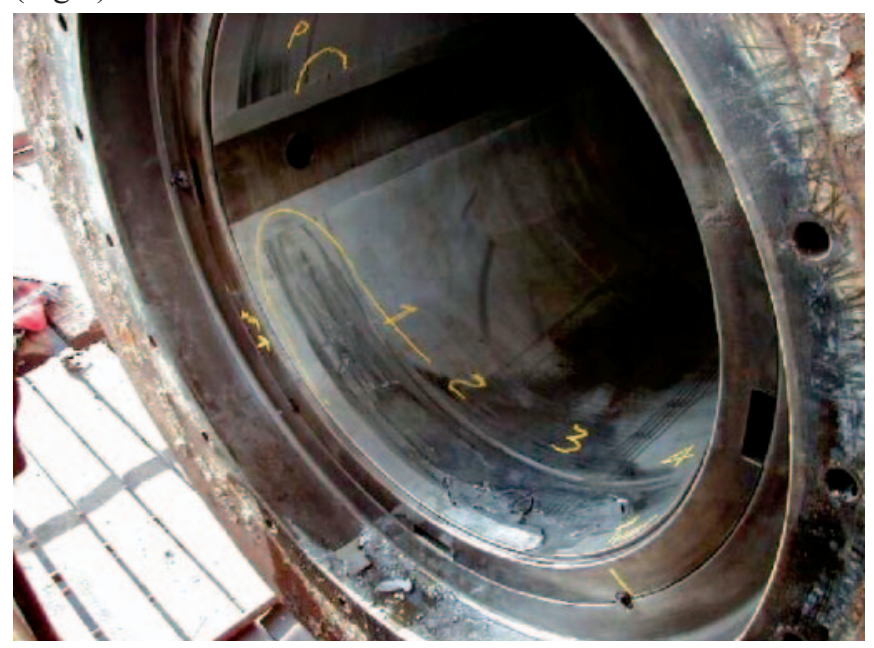

Fig. 7. A white metal bush of oil-lubricated bearing, damaged due to edging

\section{AIM OF THE RESEARCH}

The research conducted for several years has been aimed at gathering knowledge on the phenomena which occur in ship main shaft water-lubricated bearings.

Owing to the investigations have been conducted so far it was managed to determine influence of many factors on features 
of hydrodynamic bearing fitted with elastic bush lubricated with a low - viscous liquid.

Influence of the following factors on bearing features was investigated:

- diameter clearance

- bush material and sliding surface state

- mounting errors, especially axis skewing

- permanent deformation of lubricating gap, resulting from progressing wear, with taking into account possible mounting errors.

Presently is conducted a research aimed at determination of influence of bush material on bearing lifetime, and estimation of progressing wear rate so as to make it possible to elaborate a computational algorithm to identify bearing lifetime and facilitate setting deadline for necessary friction node regeneration.

\section{EXPERIMENTAL AND THEORETICAL INVESTIGATIONS}

The research on ship shaftline bearings has been conducted by Faculty of Ocean Engineering and Ship Technology, Gdańsk University of Technology, for almost ten years. Today three different test stands are applied to the experimental investigations [6]. They provide wide research possibilities, and furthermore, their dimensions make the full-scale testing of bearings possible.

It is possible to record values of resistance to motion, shaft axis trajectory, lubricating film pressure, and such parameters of lubricating medium as temperature, flow rate and pressure,

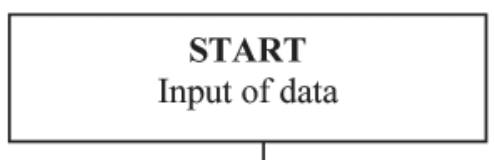

Calculations of hydrodynamic pressure field acc.

hydrodynamic lubrication theory based on Reynolds equation without bush deformation

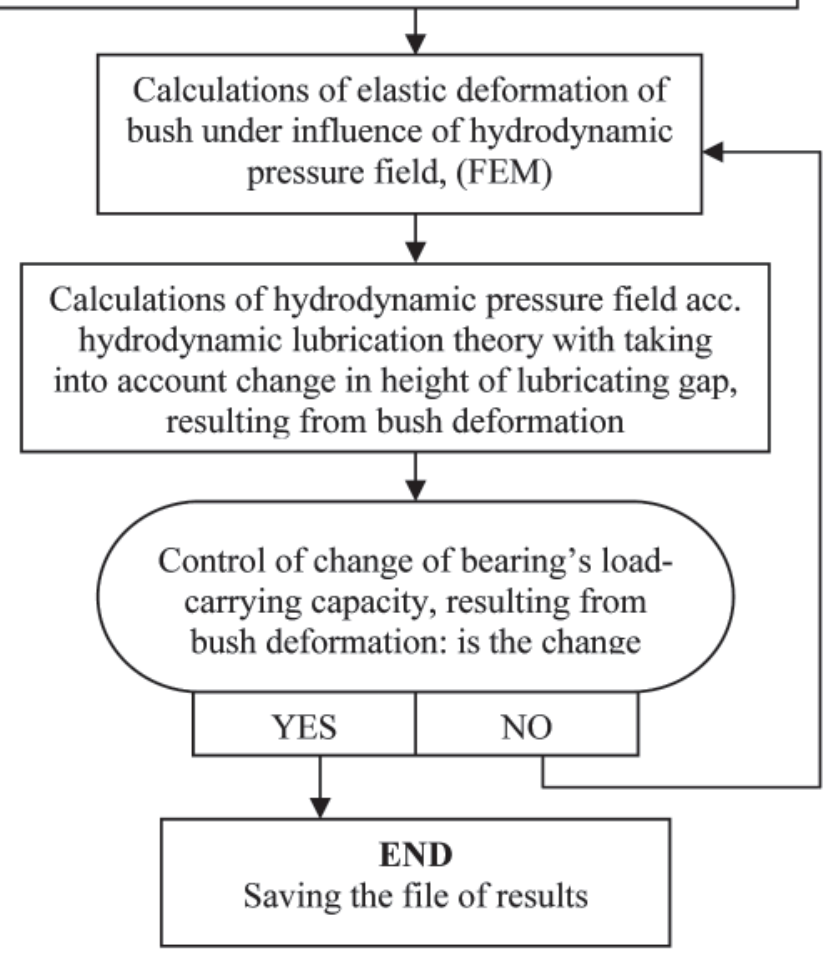

Fig. 8. Schematic diagram of the calculation method all in function of set changeable working conditions such as shaft rotational speed and load.

Apart from the experimental tests the teoretical research is also conducted. In this paper are presented calculation results obtained by using the author's original software based on hydrodynamic lubrication theory applicable to elastic bush material (EHL). The software is composed of two modules: the program based on hydrodynamic lubrication theory, written in FORTRAN language, and the module based on the finite element method (FEM) in the form of macro to a commercial software. Schematic diagram of the calculation method is presented below (Fig. 8). The calculations are performed in an iterative mode. Generally, a satisfactory result can be obtained after twenty iterations at the most (Fig. 9). Problems with achieving correct results appear in the case of extreme conditions of operation with very thin lubricating films.

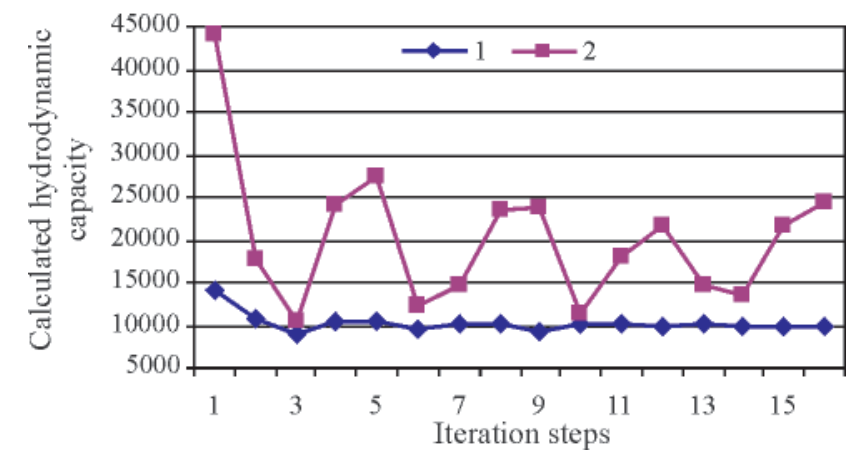

Fig. 9. Diagrams of calculated values of load-carrying capacity of a bearing in function of number of successive iteration steps: $\mathbf{1}^{\text {st }}$ series - stable solution was achieved; $2^{\text {nd }}$ series - a convergent result was not achieved

The calculations were performed for the isothermal model. As results from the temperature distribution calculations (Fig.10) and test-stand measurements the rise of lubricating film temperature is very low and amounts to less than $5^{\circ} \mathrm{C}$ usually. Therefore in the calculations the minimum change of viscosity of lubricating medium as well as of bush material properties, resulting from local rise of temperature, may be neglected.

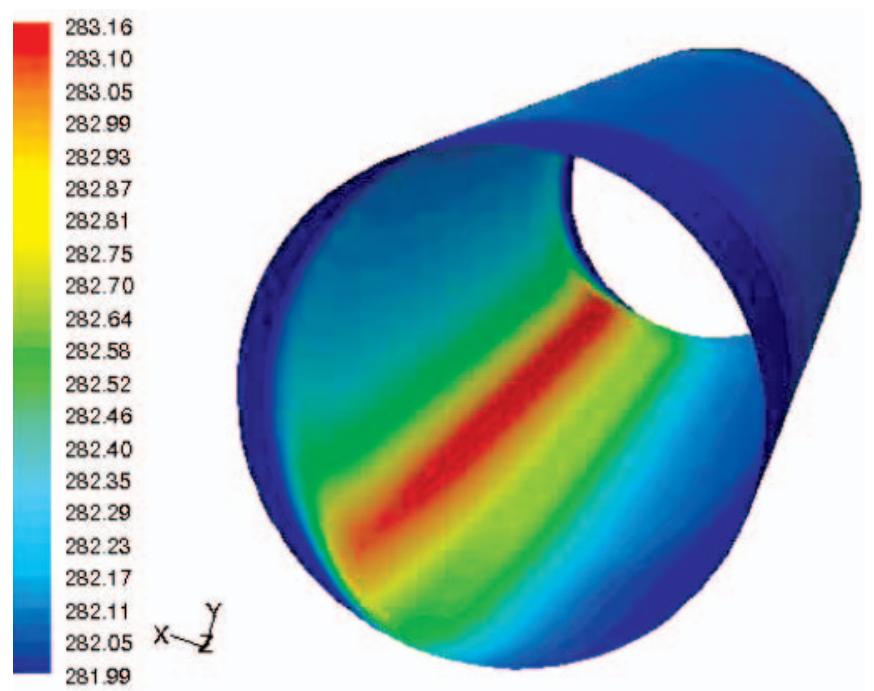

Fig. 10. Temperature distribution in lubricating film of a water-lubricated bearing, calculated by using a commercial software

Results of the calculations were verified experimentally. They confirmed that the applied calculation method is correct. It is rather hard to estimate calculation error as to accurately measure lubrication gap height is deemed questionable. In the author's opinion the calculation error does not exceed $20 \%$. 
Results of calculated and measured hydrodynamic pressure distributions in a water-lubricated bearing, are presented below (Fig. 11.)

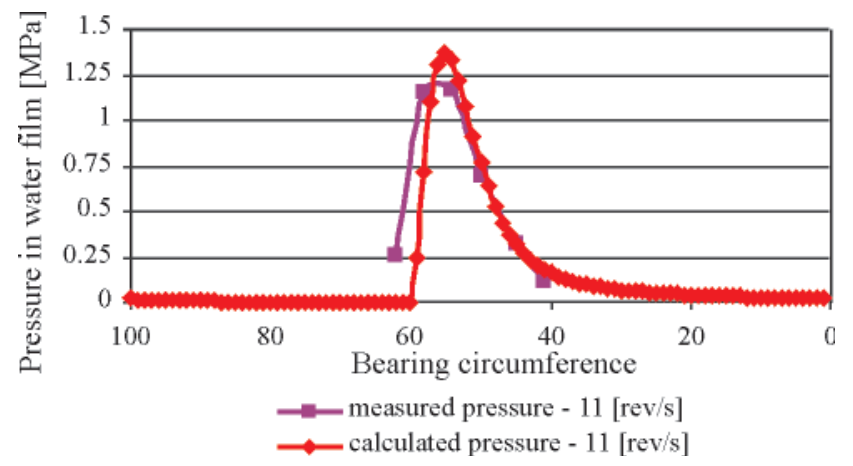

Fig. 11. Comparison of results of pressure field measurements and calculations

\section{INFLUENCE OF CLEARANCE VALUE AND BUSH MATERIAL ON BEARING QUALITIES}

Diameter clearance is of crucial impact on slide bearing qualities. The tested bearing achieves usually its largest hydrodynamic load-carrying capacity at the relative clearance value in the range from 0,001 to 0,002 (Fig. 12). However such value would be hardly applicable in practice. It should be remembered that in the typical case a completely finished polymer slide bush is seated by using shrinkage method. Therfore it is usually hard to achieve a precise value of bush inner diameter hence also a precise value of diameter clearance after seating the bearing. An additional problem is water absorption, typical for most polymers. Bush material swells as a result of the process. Because the bush is seated in a steel housing all the swelling of bush material makes bearing inner diameter smaller, which leads to significant risk of clenching the bush onto shaft and bearing blocking. It creates serious danger and therefore bearing manufacturers recommend as a rule to increase bearing gap.

An event of the kind has happened in the past during the tests conducted on one of the test stands. In effect, dismounting the bearing has taken a few days. If such situation happens on a real ship its consequences will be rather unpredictable.

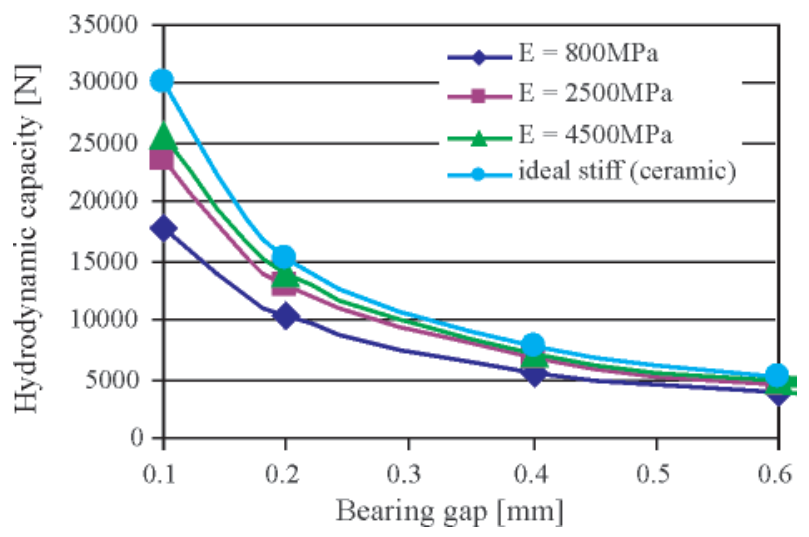

Fig. 12. Influence of bearing diameter clearance (bearing gap) and rigidity modulus of bush material on maximum load - carrying capacity of the bearing; shaft diameter of $100 \mathrm{~mm}$, bush length of $400 \mathrm{~mm}$

From the performed calculations it results that rigidity modulus greatly impacts load-carrying capacity of highloaded slide bearings (Fig. 12). It is associated with elastic deformation of bush material under hydrodynamic pressure

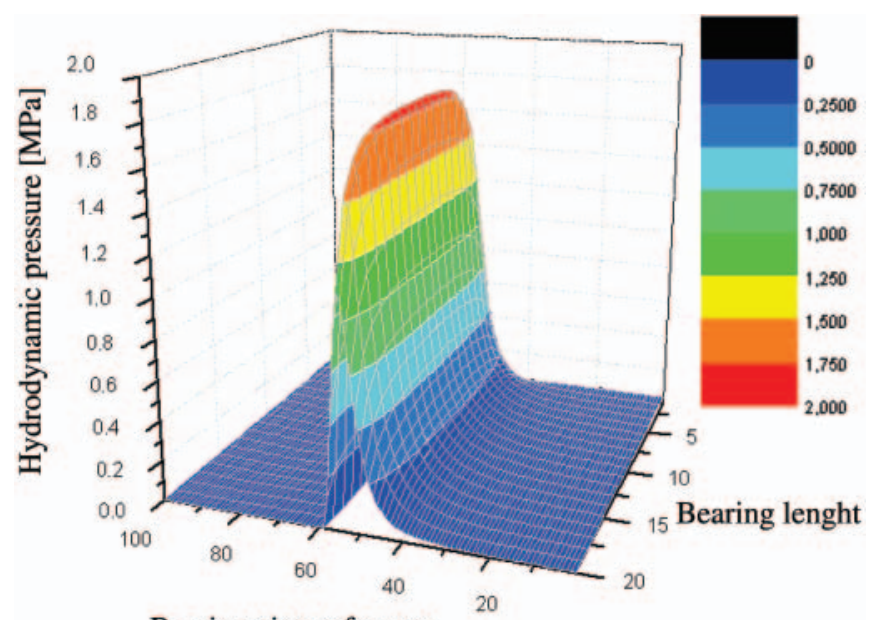

Bearing circumference

Fig. 13. Diagram of the calculated pressure field in the bearing fitted with perfectly rigid bush

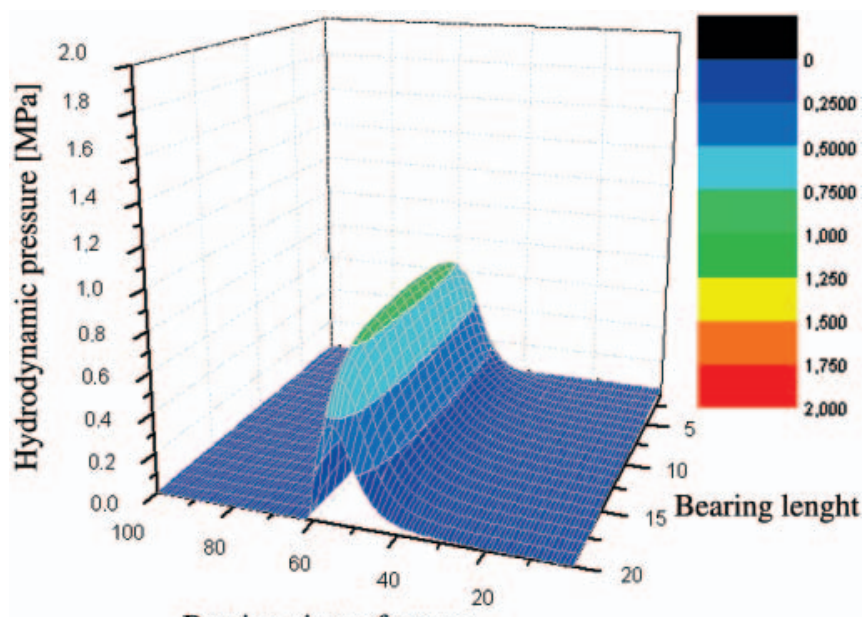

Bearing circumference

Fig. 14. Diagram of the calculated pressure field in the bearing fitted with elastic bush of $800 \mathrm{MPa}$ material rigidity modulus

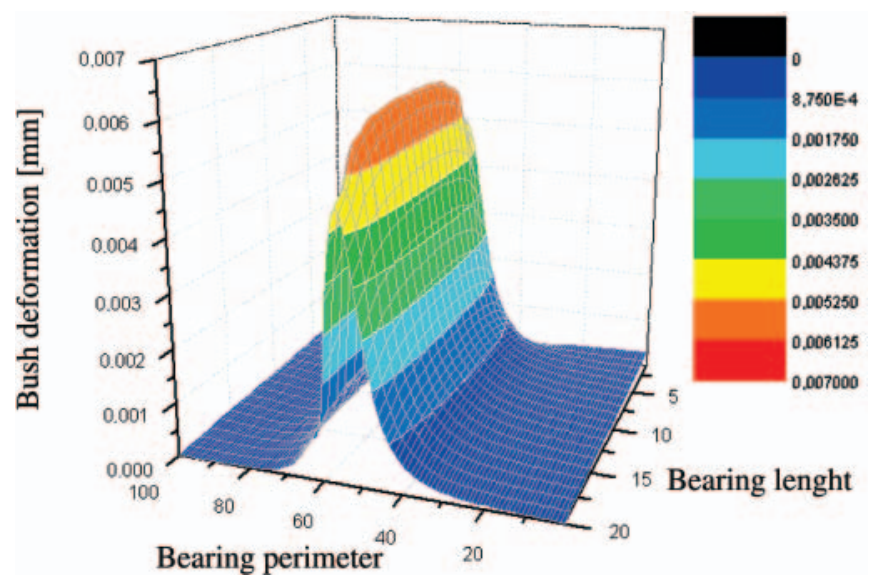

Fig. 15. Diagram of the calculated deformation field in the elastic bush of 800 MPa material rigidity modulus

of lubricating film. In effect the largest deformation takes place in the middle part of the bearing, and only slight ones - near its edges. For this reason in the central part of the bearing the lubricating gap height becomes larger and, in consequence, load-carrying capacity of the bearing - lower. (Figs. 13, 14, 15).

In the diagrams the calculated pressure field of a highloaded bearing with $5 \mu \mathrm{m}$ lubricating gap height, is presented. 
Attention should be paid to the fact that only the central part of the bush has been deformed. Therefore the smallest value of lubricating film thickness has occurred at the bearing's edges. In the case of increasing load specific situation happens in bearing of the kind: its central part operates in fluid lubrication regime but in its near-edge zones mixed friction appears and, in consequence, progressing wear process develops. Occurence of the phenomenon has been confirmed by the performed experimental tests.

\section{INFLUENCE OF BUSH SURFACE STATE ON BEARING QUALITIES}

In the trybology-matter literature many papers dealing with influence of roughness height and distribution on hydrodynamic qualities of bearing, can be found. Slide surface roughness is of a special importance for water-lubricated bearings working under fluid friction regime since values of lubricating film thickness are usually small in them. Shaft journal surface is usually grinded hence its smoothness is high. Then to machine elastic bush is more difficult. Diameter turning operation usually results in a value of roughness height amounting even to a few micrometers.

The experimental tests in question were performed for a few kinds of bush material as well as various clearance values. The bush was turned to achieve roughness direction paralell to sliding direction. A problem was to obtain the roughness having direction perpendicular to rotation direction. Therefore a machining method was elaborated similar to pull-broaching. Through a preliminarily machined opening in the bush were introduced purpose-designed cutting discs of successively larger diameters to perform bush pull-broaching. (Fig.16).

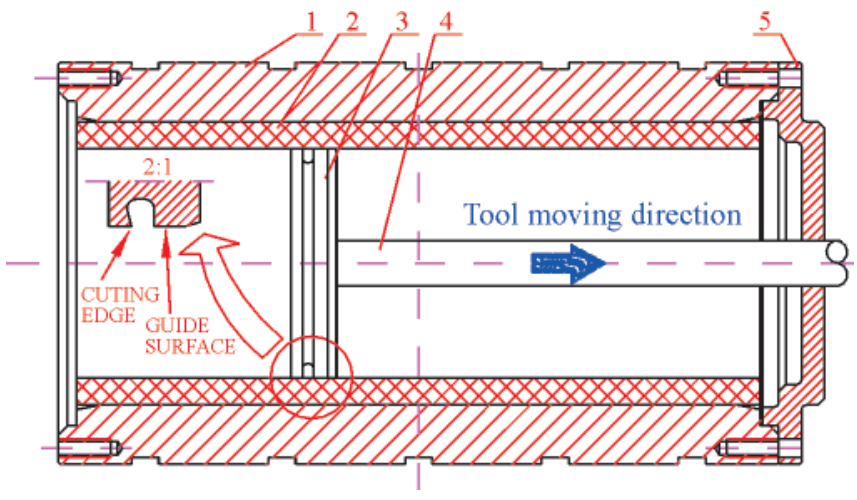

Fig. 16. Internal pull-broaching of polymer bearing bush. 1 - steel sleeve, $\mathbf{2}$ - polymer bearing bush, $\mathbf{3}$-form tool, 4 - pulling rod, 5 -cutting disc

Due to longitudinal motion of the tool roughness arrangement perpendicular to sliding direction was achieved. However in practice it turned out that certain materials, e.g. composites appeared hardly machinable as they deformed elastically. Such polymers, like polyethylene or polyamide, were less troublesome during pull-broaching operation. Despite setting the minimum thickness of cutting layer surface quality after pull-broaching operation was distinctly lower than that after turning.

The performed tests showed that roughness topography, i. e. its arrangement has not any significant impact on bearing qualities [7]. Below, an example comparison of the experimentally recorded shaft axis trajectories, is shown. The observed very small differences in the journal's position demonstrate that the state of surface has negligible effect on hydrodynamic bearing qualities. Worth adding that the tests were performed just after mounting the bearing and before bush material running-in.
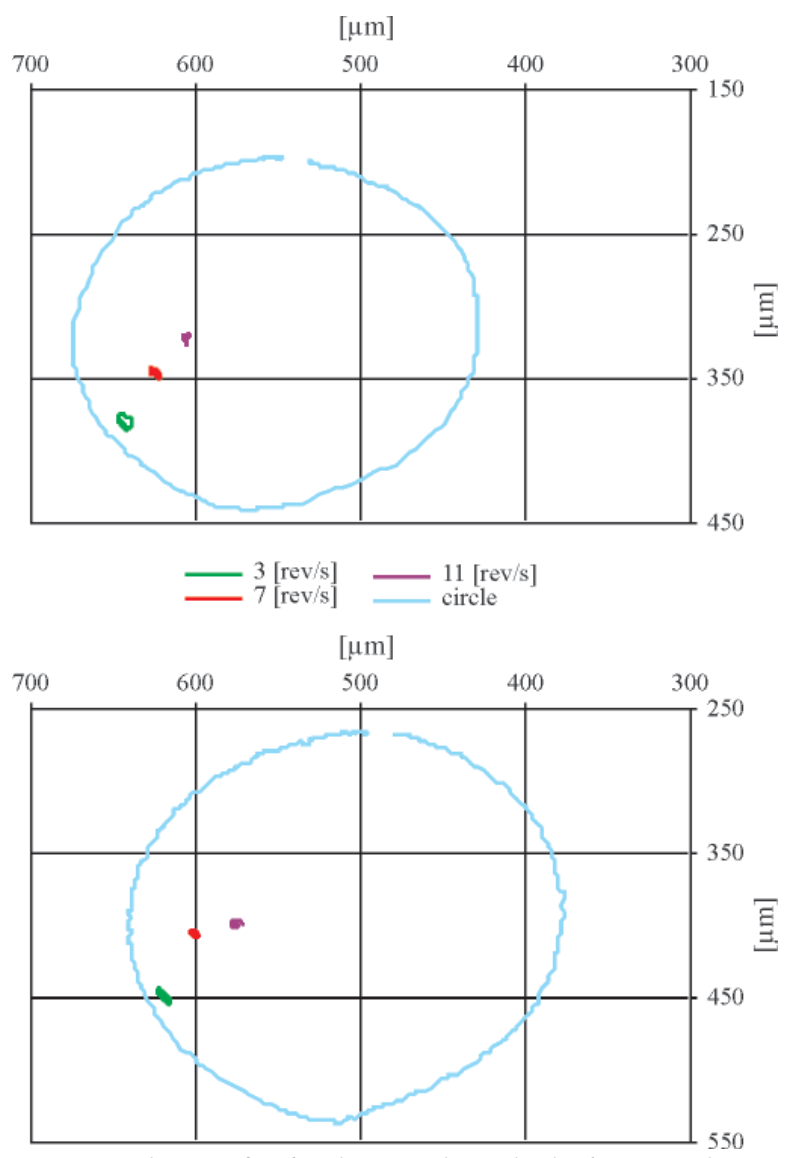

Fig. 17. A bearing fitted with PET polymer bush of 0.0027 relative clearance value; up - the bearing with turned bush; down - the bearing with pull-broached bush
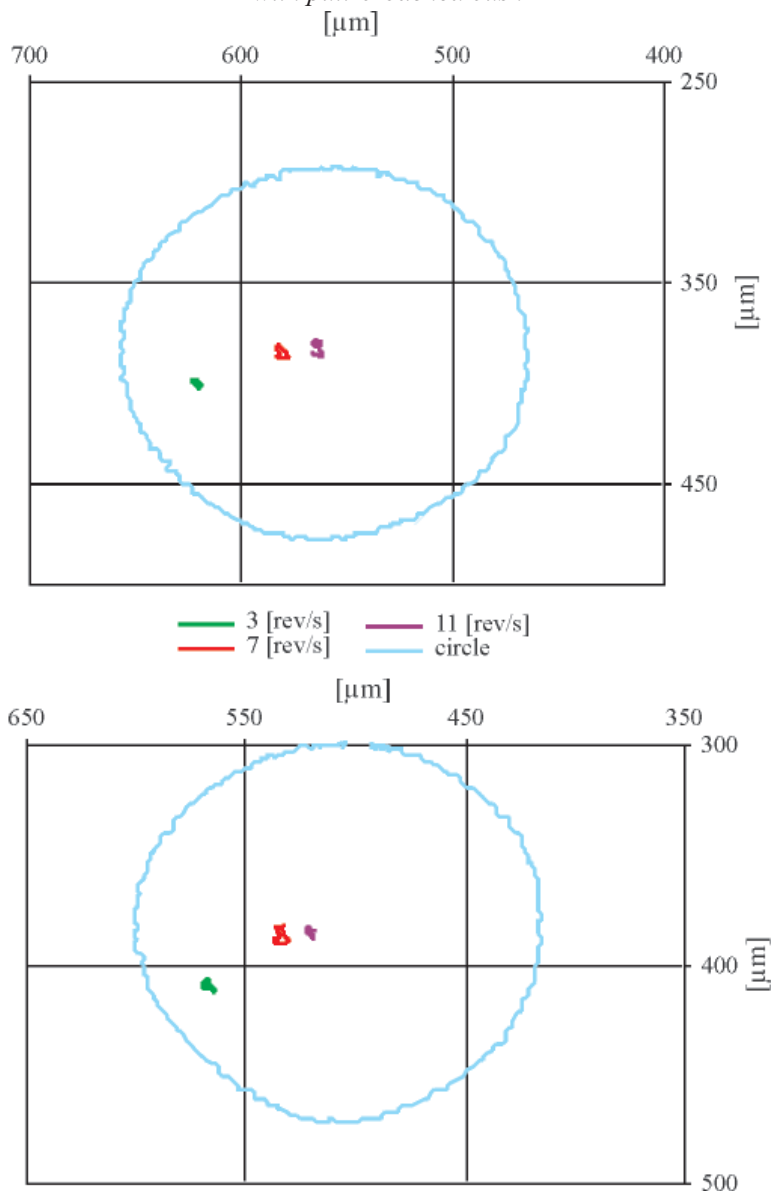

Fig. 18. Bearing fitted with composite bush of 0,0017 relative clearance value; up - the bearing with turned bush; down - the bearing with pullbroached bush 


\section{INFLUENCE OF MOUNTING ERRORS, ESPECIALLY SHAFT AXIS SKEWING, ON BEARING LOAD - CARRYING CAPACITY}

The skewing of shaft axis against bush axis produces serious danger to bearing. It may appear as a result of the improper machining and mounting of stern tube components. Such phenomenon occurs from time to time also in the case when ship hull deforms elastically during ship loading or sailing in heavy seas. Ships with long shaftlines, e.g. two-propeller double-ended ferries with amidship-located machinery room, are especially exposed to such phenomenon.

The shaft axis skewing leads to decreasing load-carrying capacity of bearing, and in extreme cases, when rigid bush materials are used, the so-called edging occurs as a rule (Fig.7). It results in an intensive local wear of bush material and sometimes also in shaft failure.

Bearings fitted with bushes made of an elastic material well tolerate non-coaxial work as they undergo large deformations and in consequence their load is distributed over an area greater than that in bearings with, for instance, a rigid composite bush.

In the below attached diagrams is presented a change of form of calculated pressure field, which results from the skewing of shaft axis. In the analyzed case the theoretically maximum value of hydrodynamic load-carrying capacity of the bearing decreased by $25 \%$ as a result of the shaft axis skewing.

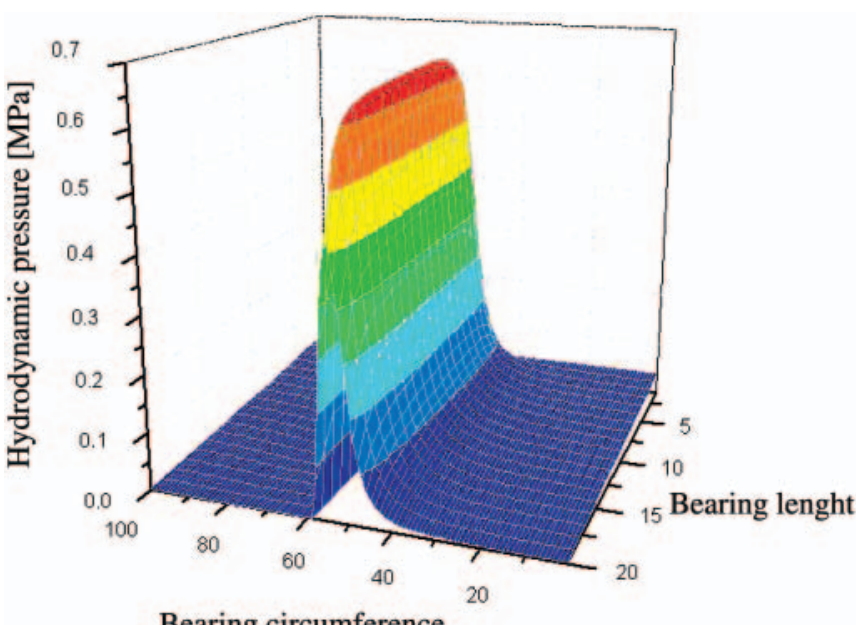

Bearing circumference

Fig. 19. Calculated pressure field in a bearing fitted with elastic bush of the material rigidity modulus $E=800 \mathrm{MPa}$

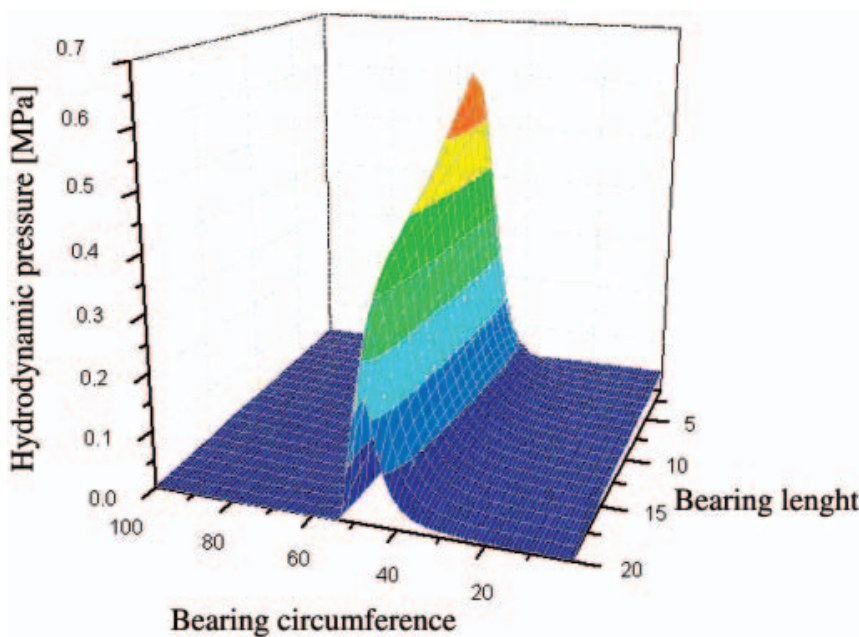

Fig. 20. Calculated pressure field in a bearing fitted with elastic bush of thematerial rigidity modulus $E=800 \mathrm{MPa}$,

and shaft axis skewed against bush

\section{INFLUENCE OF BUSH FORM ERRORS AND PROGRESSING WEAR ON BEARING LOAD - CARRYING CAPACITY}

Bearing operation performed not always in optimum conditions as well as designing and mounting errors usually lead to fast progressing wear of bush material. Consequently, it is hard to predict which form of bush would be finally obtained. From an analysis of experimental tests with many bushes as well as experience from their long-lasting operation on ships it results that bush loses its initial cylindrical form usually due to progressing wear. It step by step obtains a form of truncated cone and undergoes ovalization. In the below given figure it can be observed that the bush ovalization resulted from its long-lasting work. In the lower part of the bush a significant material wastage occurred and the bush axis was displaced by Ow value.

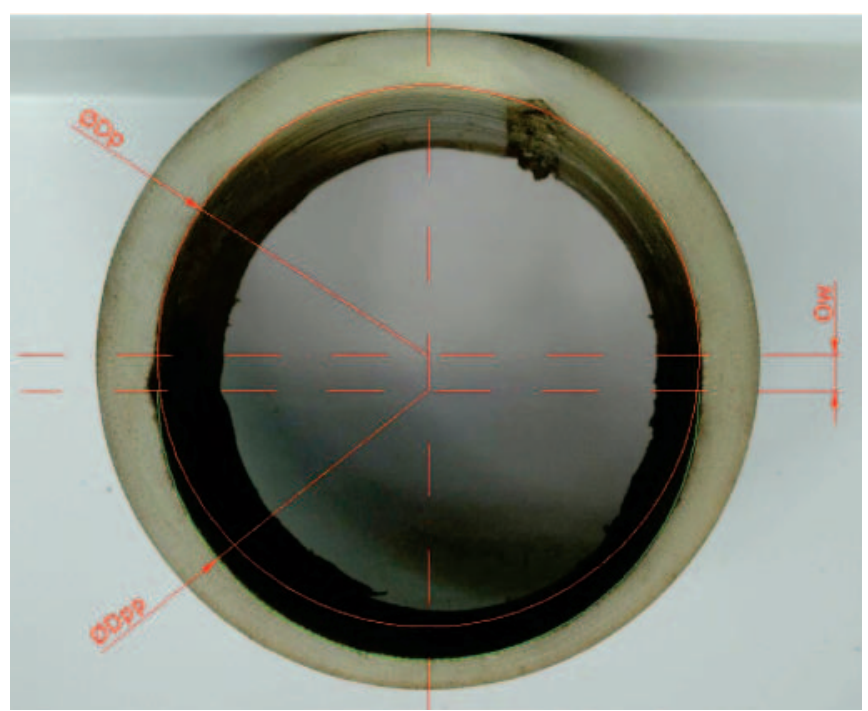

Fig. 21. Sliding bush of a water-lubricated bearing subjected to long-lasting operation which resulted in a significant wastage and associated ovalization of the bush

In practice, during ship's repair very often takes place a conflict between the shipowner who tends to limit extent of repair work and the classification society which surveys the work. For example, repair of $385 \mathrm{~mm}$ diameter stern tube bearing was given up despite $5 \mathrm{~mm}$ cylindricity errors of the journal and almost $10 \mathrm{~mm}$ change of bush diameter found in this case. Diameter clearance value for a bearing as large as that should be smaller than $1 \mathrm{~mm}$, but the really measured was greater than $10 \mathrm{~mm}$.

\section{SUMMARY}

The contemporary computational tools make it possible to relatively exactly calculate slide bearing parameters. The available software based on hydrodynamic lubrication theory or other computational methods of fluid mechanics (CFD) taking into account elastic bush deformations calculated by using the finite element method (FEM), makes it possible to conduct experiments and bearing optimization in virtual space. Ship shaft water-lubricated bearings fitted with polymer bush are sensitive units. Because of small thickness values of lubrication film their work in mixed friction regime takes often place. In the case of an erroneous position of shaft axis wear process is often fast developing in such bearings. Nonetheless they are commonly applied to smaller ships owing to their low price, simple design as well as lack of hazard to potential pollution to the environment. 
In present, a variety of sliding materials approved for shipbuilding applications can be found on the market. Most of them are products of high quality, rather insensitive to designing and mounting errors and exposure to such agressive lubricating medium as sea water.

\section{BIBLIOGRAPHY}

1. Lloyd's Register: List of materials approved for application to water-lubricated shaftline bushes on ships (in Polish)

2. Olszewski A.: Ceramic transverse bearing of conformal sliding surfaces (in Polish). Doctoral thesis, Mechanical Faculty, Gdańsk University of Technology, 2002

3. Dymarski C., Litwin W.: An experiment-based attempt to assessment of influence of surface roughness on operation of hydrodynamic water-lubricated bearing fitted with polymer bush (in Polish). The Conference , Inżynieria Łożyskowania 2007 ” (Bearing Engineering 2007),Tribologia, 2007

4. Dymarski C., Litwin W.: Influence of surface roughness of bearing bush on properties of water lubricated main shaft bearings - experimental tests. Conference NORDTRIB 2008, Tampere, Finland, 2008
5. Litwin W.: Calculations of water lubricated main shaft bearings - EHL model. Conference NORDTRIB 2008, Tampere, Finland, 2008

6. Litwin W.: The test stands for water lubricated marine main shaft bearings. NORDTRIB 2006, Helsingor, Denmark, 2006

7. Litwin W., Dymarski Cz.: Influence of surface roughness of bearing bush on properties of water lubricated main shaft bearings - experimental tests. Conference NORDTRIB 2008, Tampere, Finland, 2008

\section{CONTACT WITH THE AUTHOR}

Wojciech Litwin, Ph. D.

Faculty of Ocean Engineering and Ship Technology

Gdansk University of Technology Narutowicza 11/12

80-952 Gdansk, POLAND

e-mail: wlitwin@pg.gda.pl 